\title{
Modal and whirling analysis of coupled lateral and torsional vibration of herringbone gear
}

\author{
Siyu Chen · Jinyuan Tang • Changjiang Zhou • Zehua Hu
}

Received: 17 July 2013 / Revised: 14 November 2013 / Accepted: 18 November 2013 / Published online: 10 December 2013

(C) Springer-Verlag Berlin Heidelberg 2013

\begin{abstract}
The coupled lateral and torsional motions are significant for the herringbone gear in high-speed applications. The present work attempts to investigate the influences of damping, eccentric mass and time varying mesh stiffness of gear pair on the modal vibration of a herringbone gear pair. Under high-speed condition, the gyroscopic performance as a result of coupled lateral and torsional motions cannot be ignored. To achieve a targeted analysis, the equivalent mesh stiffness of the herringbone gear pair is calculated in preprocessing based on the finite element method by considering the thin rim and web. Subsequently, an analytical model for coupled lateral and torsional motions of the herringbone gear is proposed. Then, the natural frequencies, synchronous whirling speed, critical speed, as well as the transient behaviors with time invariant and time varying stiffness, are calculated numerically. The results in Campbell diagram show that the damping affects the critical speed slightly while the eccentric mass will reduce the critical speed significantly. Transient dynamics analysis shows that no matter which stiffness models are used, the high frequency components are predominant, which may be the results of frequency veering phenomena at high order natural frequency. The present work indicates the necessity of paying attention to the critical speed relative to mesh frequency in high speed gear applications.
\end{abstract}

Keywords Herringbone gear - Modal vibration - Whirling · High speed

S. Chen $\cdot$ J. Tang $(\bowtie) \cdot$ Z. Hu

State Key Laboratory of High Performance Complex

Manufacturing, Central South University, Changsha 410083,

Hunan, China

e-mail: jytangcsu_312@163.com

C. Zhou

State Key Laboratory of Advanced Design and Manufacture for Vehicle Body, Hunan University, Changsha 410082, China

\section{List of Symbols}

\begin{tabular}{|c|c|}
\hline$\alpha_{i}$ & $(i=p, g)$ Angular perturbation \\
\hline$\alpha_{n}$ & Normal pressure angle \\
\hline$\alpha_{1}$ & Supporting damping coefficient \\
\hline$\beta$ & Helix angle \\
\hline$\beta_{1}$ & Supporting damping coefficient \\
\hline$\theta$ & Rotation displacement \\
\hline$\omega_{i}$ & Rotation velocity \\
\hline$\delta_{m}$ & Mesh deflection \\
\hline$\lambda$ & eigenvalues \\
\hline$\zeta$ & Damping ratio \\
\hline$\tau$ & Dummy integration variable \\
\hline$v$ & Poisson ratio \\
\hline$\Omega_{i}$ & Angular velocity \\
\hline$c_{m}$ & Mesh damping \\
\hline$e_{i}$ & Eccentric length \\
\hline$k_{m}$ & Mesh stiffness \\
\hline$m$ & Normal module \\
\hline$m_{i}$ & mass \\
\hline$r_{b i}$ & Basic radius of gear \\
\hline$x, y$ & Translation displacement \\
\hline$z_{i}$ & Number of teeth \\
\hline$E$ & Young's modulus \\
\hline $\mathrm{T}$ & Function of kinetic energy \\
\hline $\mathrm{U}$ & Function of strain energy \\
\hline$T_{i}$ & Drag torque \\
\hline$J_{P i}$ & Moment of inertia \\
\hline $\mathrm{X}, \mathrm{Y}, \mathrm{Z}$ & Unit vector of axis \\
\hline $\mathbf{M}_{i}$ & Mass matrix \\
\hline $\mathbf{G}_{i}$ & Gyroscopic matrix \\
\hline $\mathbf{C}$ & Damping matrix \\
\hline$\tilde{Q}_{i}$ & Nonlinear force \\
\hline $\mathbf{K}$ & Stiffness matrix \\
\hline $\mathbf{q}$ & Variation vector \\
\hline
\end{tabular}




$\begin{array}{ll}\mathbf{F}_{i n} & \text { Internal excitation vector } \\ \mathbf{F}_{e x} & \text { External excitation vector } \\ \text { SWL } & \text { Synchronous whirl line } \\ \text { TR } & \text { Torsional response } \\ \text { LR } & \text { Lateral response } \\ \text { TE } & \text { Transmission error } \\ \text { NLTE } & \text { No load transmission error }\end{array}$

\section{Introduction}

In simple gear dynamic models, the effects of shaft and bearing flexibility are simplified or neglected with an underlying assumption, to study the dynamic characteristics along the mesh plane. Theoretically, the coupled lateral and torsional vibration characteristics may be different from those obtained in an uncoupled analysis model [1-5].

Therefore, the coupled effects of lateral and torsional vibrations have attracted increasing attention in gear dynamics analysis. For example, in high-speed applications, the gear dynamic force is transmitted through the shaft and bearing. Conversely, the shaft deflection, together with bearing stiffness and damping may affect the mesh characteristics of a gear pair. Kang and Kahraman's [6] experimental results show that the shaft flexibility can alter the dynamic response of a gear pair. Furthermore, the coupled lateral and torsional modes are also confirmed experimentally in a planetary gear system [4].

Dynamic models combining gears with flexible shafts and bearings have been investigated by numerous scholars. The core concept of their methods is to represent shaft by a finite element model and introduce bearing flexibility by a linearized 8-coefficient bearing model. The stiffness and damping contained in the shaft and bearing exert significant influence on the modal behavior [7]. Lund [8] first considered coupled effects in the torsional-lateral vibrations for a geared rotor system. Iida et al. [9] considered a simple geared system with coupled torsional and flexural vibrations. Kahraman et al. $[2,10]$ developed a gear rotor system model, in which a finite element model is used to represent shaft and the flexibility of bearing is considered. Baud and Velex [11] investigated the dynamics of gear-shaft-bearing systems using a nonlinear gear model. A nonlinear approach, proposed by Baguet and Velex [12], is applied to analyze the dynamic behaviors of the gear-shaft-bearing model. The shaft is also represented by a finite element model and the proposed gear element can account for time varying mesh stiffness as well as tooth shape deviations. Subsequently, Baguet and Jacquenot [13] extended the model to helical gears and finite-length hydrodynamic bearing systems. Kang et al. [14] investigated the dynamic behaviors of a gear-rotor system with effects of viscoelastic supports, gear eccentricity, transmission error, and residual shaft bow.
It is well known that a common source of rotor whirling is rotor unbalance, since real rotors can seldom be perfectly balanced in practice. On the other hand, for a gear rotor system, the mesh force will push away the contact pair from the static rotation axes and thus the gyroscopic performance can not be ignored. As modal analysis with gyroscopic effect is intractable [3,15], under some conditions, the gyroscopic force of a gear rotor system is neglected [16,17]. However, in high-speed applications such as in turbofan and turboprop engines, the gyroscopic effect should be taken into consideration as stated by Abousleiman et al. [18]. Kumar and Rao $[19,20]$ studied the whirling motion and critical speed of a pair of spur gears by considering the gyroscopic effect.

The herringbone gear, also named double-helical gear, as the main component of a power flow transmission system, has been widely used in aeroengine operating under high speed and high power conditions [21,22]. It has unique characteristics of large contact ratio, smooth transmission and low noise, which make the system meet the requirements of heavy duty working conditions. Additionally, the herringbone gear can accommodate the axial forces which may result from axial misalignment and reduce the rigorous request for bearing. However, literature on the herringbone gear is quite rare and the corresponding analysis is limited to geometrical analysis, and load distribution and transmission error under static conditions. Jauregui and Gonzalez (cited in Ref. [23]) have studied the axial vibrations in herringbone gears by using a single degree-of-freedom dynamic model. Ajmi and Velex [23] presented a model for analyzing the quasi-static and dynamic behaviors of the herringbone gear by taking into account time varying mesh stiffness, gear distortion and shape modifications. In their model, the herringbone gears were constructed by two identical gear elements with opposed helices separated by Euler beam elements. The gyroscopic effect is ignored and mesh stiffnesses are not independent since they are all connected to two deformable shafts.

Theoretically, the mesh stiffness of herringbone gears can be calculated by using the method adopted in helical gears [24]. But the thin rim and web for reducing the total mass of the transmission system will disturb this strategy in practice.

The main motivations of the present paper are to reveal the modal vibration structure of high speed herringbone gears with gyroscopic effects. First, the equivalent mesh stiffness of the herringbone gear pair with thin rim and web is calculated based on the finite element method. Then, a dynamic model including five rigid body degrees of freedom of each gear (the axial degree of each gear is neglected) is developed to analyze modal vibrations. Finally, the whirling motion and critical speed of the herringbone gear is analyzed numerically. 


\section{Mesh stiffness of herringbone gear}

To study the dynamic mesh performance of a pair of herringbone gears, the mesh stiffness and static transmission error should be first calculated. Generally, the mesh stiffness of a herringbone gear can be calculated by using the method adopted for helical gears, where the left and right helical gears are connected by using Euler type finite beam elements [23]. In order to account for the effects of the thin web and rim of the herringbone gear, a calculation method for equivalent mesh stiffness based on the finite element method is proposed in the present work.

With the basic design parameters of the herringbone gear in Table 1, the finite element model is constructed as shown in Fig. 1. In this model, only nine pairs of teeth are modeled, assuming that the effects of other pairs are negligible. The

Table 1 Basic design parameters of Herringbone gear

\begin{tabular}{|c|c|c|c|}
\hline & Symbol & $\begin{array}{l}\text { Pinion } \\
(i=p)\end{array}$ & $\begin{array}{l}\text { Gear } \\
(i=g)\end{array}$ \\
\hline Number of teeth & $z_{i}$ & 23 & 157 \\
\hline Normal pressure angle $\left({ }^{\circ}\right)$ & $\alpha_{n}$ & 20 & \\
\hline Normal module (mm) & $m$ & 3.0215 & \\
\hline Helix angle $\left(^{\circ}\right)$ & $\beta$ & 30 & \\
\hline Young's modulus (MPa) & $E$ & 210,000 & \\
\hline Poisson ratio & $v$ & 0.3 & \\
\hline Mass (kg) & $m_{i}$ & 2.16 & 41.25 \\
\hline \multirow[t]{2}{*}{ Moment of inertia $\left(\mathrm{kg} * \mathrm{~m}^{2}\right)$} & $J_{P i}$ & 0.0036 & 0.97 \\
\hline & $J_{D i}$ & 0.0025 & 1.91 \\
\hline \multirow[t]{6}{*}{ Supporting stiffness (N/m) } & $k_{x i}$ & $6.53 \mathrm{E} 7$ & 4.16 E7 \\
\hline & $k_{y i}$ & $6.53 \mathrm{E} 7$ & 4.16 E7 \\
\hline & $k_{\theta_{x i}}$ & $6.16 \mathrm{E} 5$ & $2.06 \mathrm{E} 6$ \\
\hline & $k_{\theta y i}$ & $6.16 \mathrm{E} 5$ & $2.06 \mathrm{E} 6$ \\
\hline & $k_{x i \theta_{y i}}$ & $2.28 \mathrm{E} 5$ & $5.56 \mathrm{E} 6$ \\
\hline & $k_{y i \theta_{x i}}$ & $2.28 \mathrm{E} 5$ & $5.56 \mathrm{E} 6$ \\
\hline \multirow[t]{2}{*}{ Supporting damping coefficient } & $\alpha_{1}$ & 100 & \\
\hline & $\beta_{1}$ & $5 \mathrm{E}-5$ & \\
\hline Mesh damping (Ns/mm) & $c_{m}$ & 200 & \\
\hline
\end{tabular}

hexahedron C3D8R element is adopted to mesh the gear teeth and body. The element number for the pinion and gear is 166,864 and 247,546 respectively. Two reference points are created, which are geometrical points that can be used in Abaqus to simulate rigid bodies. The drag torque is applied to the gear and the rotation displacement is added to the pinion. The Abaqus/standard procedure is performed to analyze the static teeth contact.

Then the transmission errors can be defined from the actual total angles of rotation as [25]

$T E=r_{b p} \alpha_{p}+r_{b g} \alpha_{g}+N L T E$

Here, $r_{b i}(i=p, g)$ is basic radius of gear $i, \tau$ is a dummy integration variable, $\alpha_{i}$ is the angular perturbation with respect to rigid-body ration at the reference point, and NLTE is no load transmission error.

Simply, the mesh process of the herringbone gear along the line of action can be assumed as an equivalent mesh stiffness and damping. In the static analysis, the damping effect is negligible and the equivalent mesh stiffness can be deduced as

$k_{m}=\frac{T_{g}}{T E \cdot r_{b g} \cos \beta}$

here, $\beta$ is helix angle, $T_{g}$ is drag torque, and $r_{b g}$ is basic radius of gear. Generally, the 3D models of a gear pair are perfect, namely $N L T E=0$. But after the gear body is partitioned and meshed, the geometric error is induced inevitably. Besides, the numerical error is also inevitable. Accordingly, NLTE must be taken into account in the mesh stiffness analysis. To overcome the two problems, the static analysis is performed under two load conditions, i.e., high load $(14,000 \mathrm{~N} / \mathrm{m})$ and no load $(10 \mathrm{~N} / \mathrm{m}$. Zero load is irregularity for the static analysis in the Abaqus. Under this condition, the contact deflection is neglected). Then the NLTE is obtained according to the Finite element analysis of herringbone under no load condition.

With a drag torque of $14,120 \mathrm{Nm}$, the mesh forces are illustrated in Fig. 2, where the abscissa axis refers to Abaqus step time without unit, and the symbol $T$ means gear mesh period. From the figure, it can be known that there are at least four pairs of teeth under mating process. The total con-

Fig. 1 Finite model of herringbone gear pair
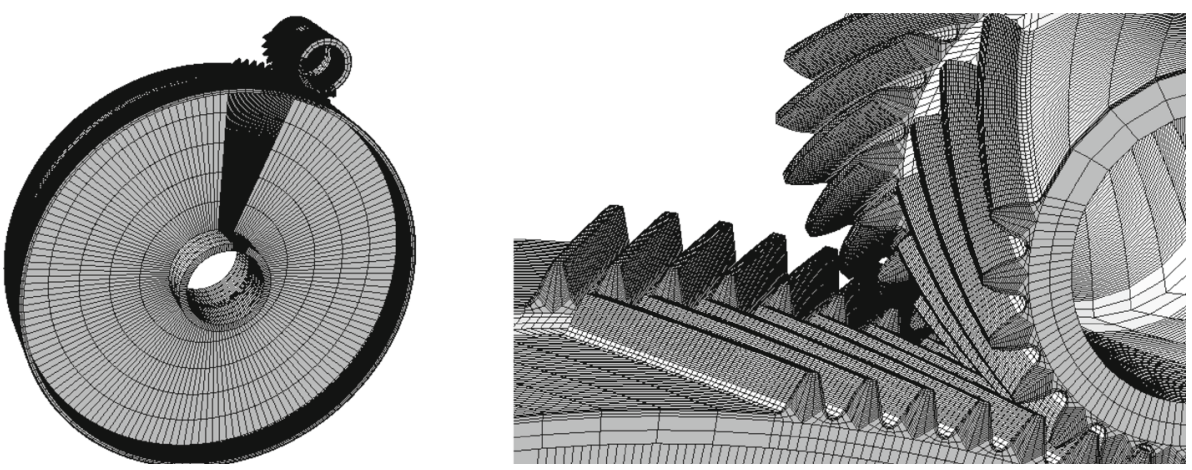
Fig. 2 Mesh force

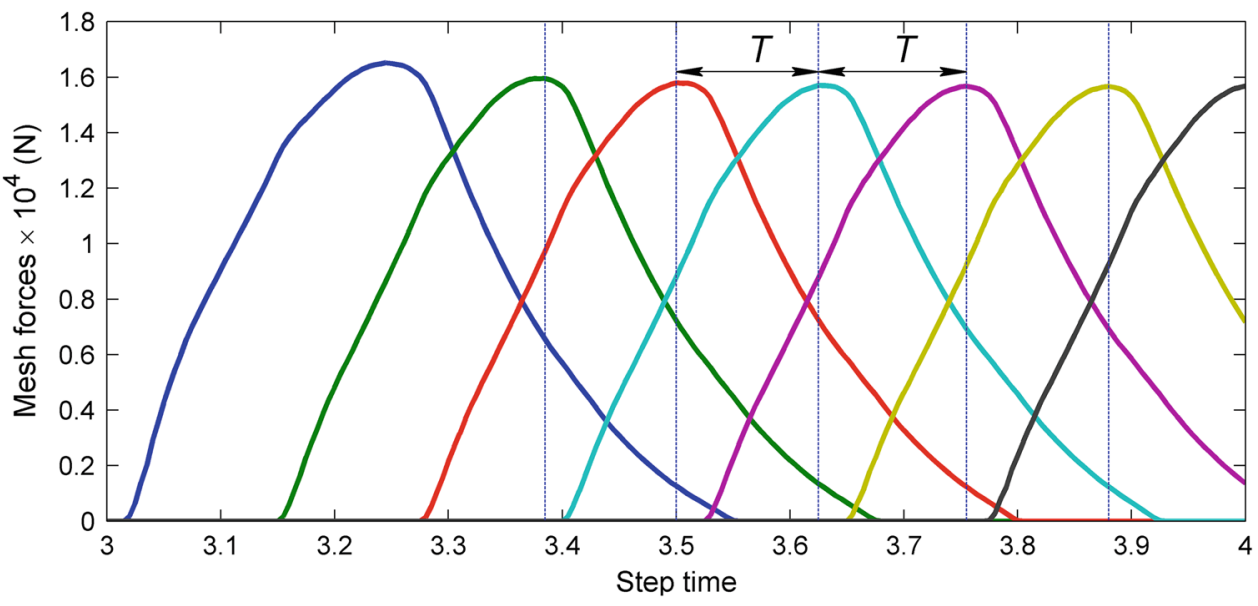

Fig. 3 Equivalent mesh stiffness of herringbone gear

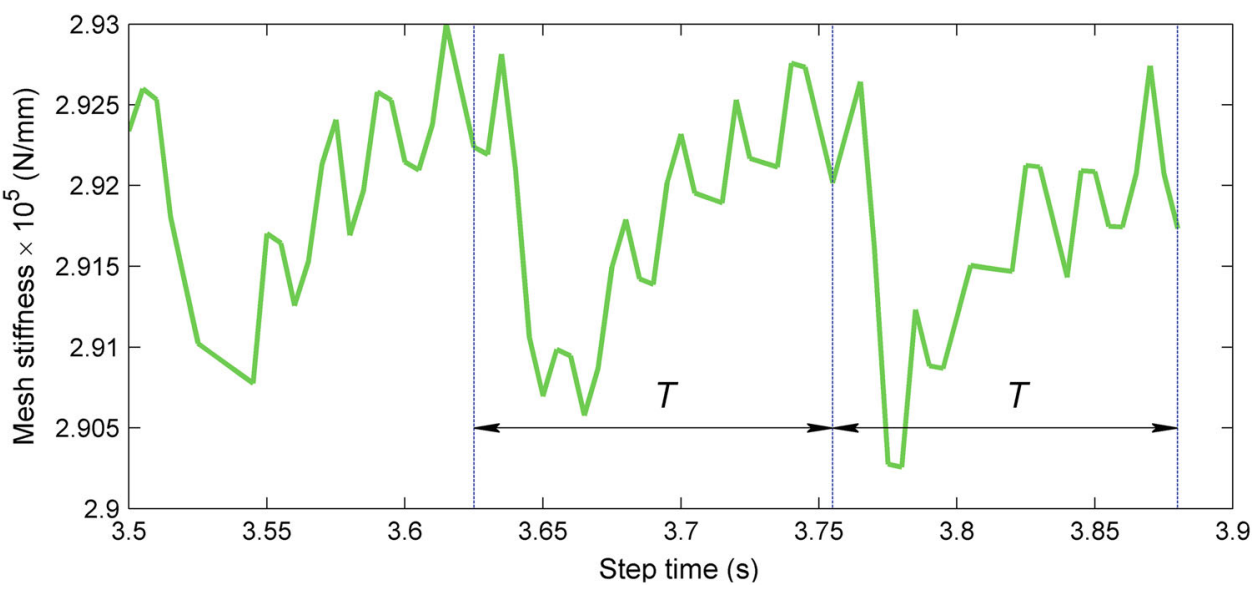

tact ratio is much larger than the theoretical value (in theory, the transverse contact ratio is 1.409 and the axial contact ratio is 1.896), which may result from the deflection of tooth and foundation. With a high contact ratio, the herringbone gear will rotate and transmit torque smoothly. With Eq. (2), the equivalent mesh stiffness is illustrated in Fig. 3, which indicates that the equivalent mesh stiffness is time periodic approximately. The mesh stiffness of the herringbone gear is different from that of other gears as there are many sharp peaks in the stiffness curve, which is mainly caused by the changing of tooth number in contact.

\section{System model}

The herringbone gear transmission system is considered to be rigid with zero axial extension to translational displacements and three rotation displacements. As mentioned in Ref. [26], it is convenient to consider two angles $\theta_{x i}(i=p, g)$ and $\theta_{y i}$ as coordinates describing the angle motion of the rotor as shown in Fig. 4. In this way, $\theta_{x i}$ becomes an angular displacement in the $y z$ plane, describing a small rotation around the $x$-axis while $\theta_{y i}$ becomes an angular displacement in the $x z$ plane describing a rotation around the $y$-axis. Here, note that the profile errors of both helical gears are not considered in the present paper, thus unlike single helical gear, they do not produce axial force.

The rotation velocity of gear $i$ can be written as

$$
\begin{aligned}
\omega_{i}= & {\left[\cos \left(\Omega_{i}+\dot{\alpha}_{i}\right) \dot{\theta}_{y i}+\sin \left(\Omega_{i}+\dot{\alpha}_{i}\right) \dot{\theta}_{x i}\right] \mathbf{X} } \\
& +\left[\sin \left(\Omega_{i}+\dot{\alpha}_{i}\right) \dot{\theta}_{y i}-\cos \left(\Omega_{i}+\dot{\alpha}_{i}\right) \dot{\theta}_{x i}\right] \mathbf{Y} \\
& +\left[\left(\Omega_{i}+\dot{\alpha}_{i}\right)+\frac{\theta_{x i} \dot{\theta}_{y i}-\theta_{y i} \dot{\theta}_{x i}}{2}\right] \mathbf{Z}
\end{aligned}
$$

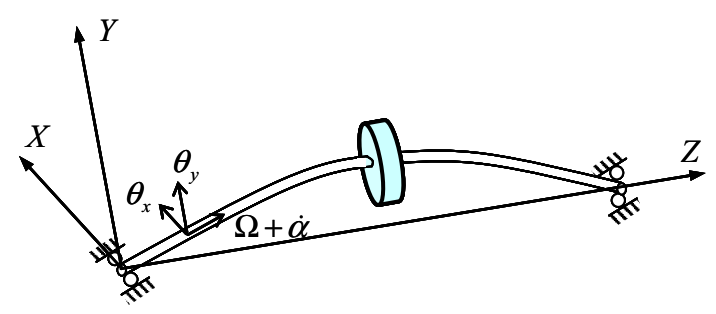

Fig. 4 Typical rotor configuration and coordinate system 
Here, $\Omega_{i}$ is spin speed and $\dot{\alpha}_{i}$ is torsional velocity. Then, the kinetic energy of gear $i$ including torsional kinetic energy and eccentricity $e$ are obtained,

$$
\begin{aligned}
T_{i}= & \frac{1}{2} m_{i}\left(\dot{x}_{i}^{2}+\dot{y}_{i}^{2}\right)+\frac{1}{2} J_{D i}\left(\theta_{y i}^{2}+\theta_{x i}^{2}\right) \\
& +\frac{1}{2} J_{P i}\left(\Omega_{i}+\dot{\alpha}_{i}\right)\left(\theta_{x i} \dot{\theta}_{y i}-\theta_{y i} \dot{\theta}_{x i}\right)+\frac{1}{2} J_{P i}\left(\Omega_{i}+\dot{\alpha}_{i}\right)^{2} \\
& +m_{i} e_{i}\left(\Omega_{i}+\dot{\alpha}_{i}\right)\left[\dot{y}_{i} \cos \left(\Omega_{i} t+\alpha_{i}+\varphi_{i}\right)\right. \\
& \left.-\dot{x}_{i} \sin \left(\Omega_{i} t+\alpha_{i}+\varphi_{i}\right)\right]+\frac{1}{2} m e_{i}^{2}\left(\Omega_{i}+\dot{\alpha}_{i}\right)^{2}
\end{aligned}
$$

The kinetic energy can be written in matrix form as

$$
T_{i}=\frac{1}{2} \dot{\mathbf{q}}_{i}^{T} \mathbf{M}_{i} \dot{\mathbf{q}}_{i}+\frac{1}{2} \dot{\mathbf{q}}_{i}^{T} \mathbf{G}_{i} \mathbf{q}_{i}+\tilde{Q}_{i}\left(\mathbf{q}_{i}, \dot{\mathbf{q}}_{i}\right)
$$

here,

$$
\begin{aligned}
& \mathbf{q}_{i}=\left[x_{i}, y_{i}, \theta_{x i}, \theta_{y i}, \alpha_{i}\right]^{T} \\
& \mathbf{M}_{i}=\operatorname{diag}\left(m_{i}, m_{i}, J_{D i}, J_{D i}, J_{P i}+m e_{i}^{2}\right)
\end{aligned}
$$

$$
\mathbf{G}_{i}=\Omega_{i}\left[\begin{array}{ccccc}
0 & 0 & 0 & 0 & 0 \\
0 & 0 & 0 & 0 & 0 \\
0 & 0 & 0 & J_{P i} & 0 \\
0 & 0 & -J_{P i} & 0 & 0 \\
0 & 0 & 0 & 0 & 0
\end{array}\right]
$$

$$
\begin{aligned}
\tilde{Q}_{i}\left(\mathbf{q}_{i}, \dot{\mathbf{q}}_{i}\right)= & +\frac{1}{2} J_{P i} \dot{\alpha}_{i}\left(\theta_{x i} \dot{\theta}_{y i}-\theta_{y i} \dot{\theta}_{x i}\right) \\
& +\frac{1}{2} J_{P i}\left(\Omega_{i}\right)^{2}+J_{P i} \Omega_{i} \dot{\alpha}_{i} \\
& +m_{i} e_{i}\left(\Omega_{i}+\dot{\alpha}_{i}\right)\left[\dot{y}_{i} \cos \left(\Omega_{i} t+\alpha_{i}+\varphi_{i}\right)\right. \\
& \left.-\dot{x}_{i} \sin \left(\Omega_{i} t+\alpha_{i}+\varphi_{i}\right)\right] \\
& +\frac{1}{2} m e_{i}^{2}\left(\Omega_{i}\right)^{2}+m e_{i}^{2} \Omega_{i} \dot{\alpha}_{i}
\end{aligned}
$$

Figure 5a shows a gear pair, in which the mesh teeth are replaced by mesh stiffness $k_{m}$ and damping $c_{m}$ along the pressure line in the action plane. As mentioned above, the axial force of the herringbone gear is zero when the teeth profile errors are neglected. Then the herringbone gears can be simplified as an equivalent spur gear pair in the mesh process, shown in Fig. 5 b.

The deflection at any potential point of contact can be expressed as

$$
\begin{aligned}
\delta_{m}= & \left(x_{p}-x_{g}\right) \cos \alpha_{n}+\left(y_{p}-y_{g}\right) \sin \alpha_{n} \\
& -\left(r_{b p} \alpha_{p}+r_{b g} \alpha_{g}\right)-e\left(\Omega_{m} t\right) \\
= & \mathbf{V}^{T} \mathbf{q}-e\left(\Omega_{m} t\right)
\end{aligned}
$$

here,

$$
\begin{aligned}
\mathbf{q}= & {\left[\begin{array}{l}
\mathbf{q}_{p} \\
\mathbf{q}_{g}
\end{array}\right], } \\
\mathbf{V}^{T}= & \left(\cos \alpha_{n}, \sin \alpha_{n}, 0,0,-r_{b p},-\cos \alpha_{n},\right. \\
& \left.-\sin \alpha_{n}, 0,0,-r_{b g}\right)
\end{aligned}
$$

Then the gear strain energy is

Fig. 5 Gear mesh model

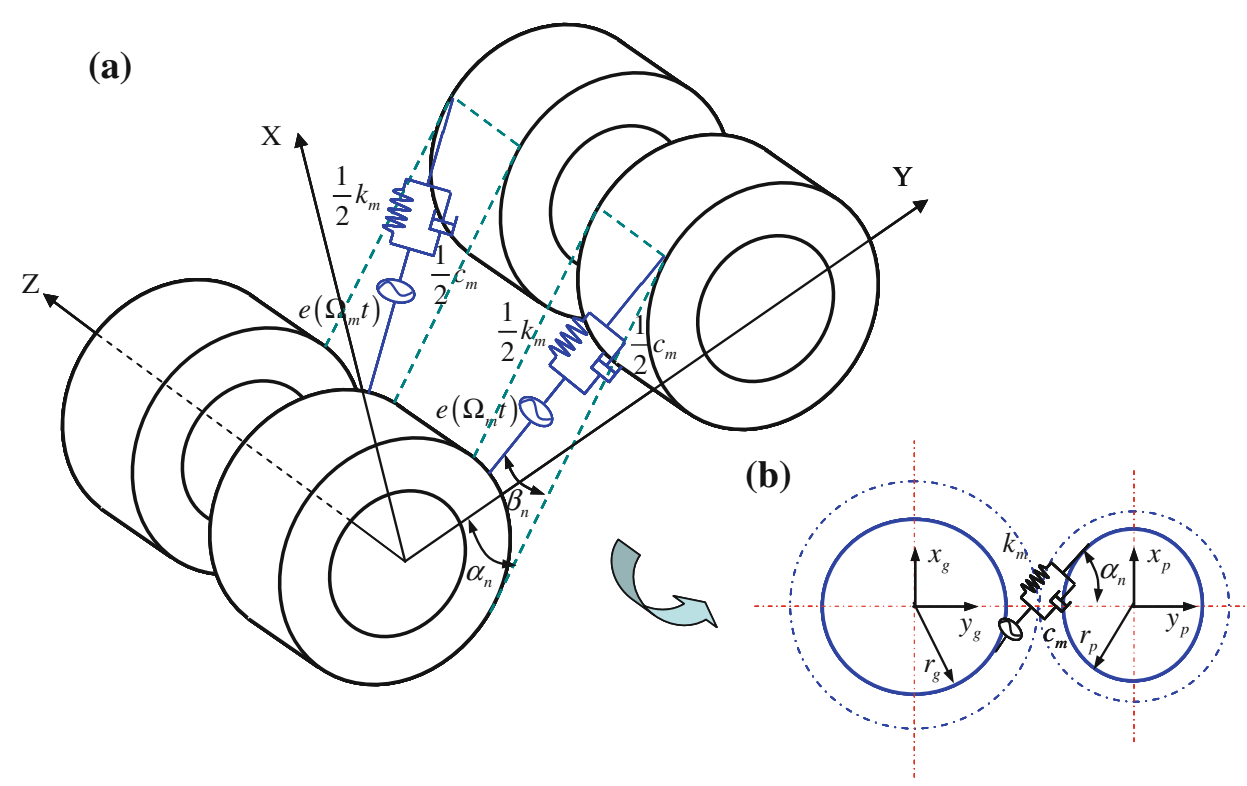




$$
\begin{aligned}
\mathrm{U}_{1} & =\frac{1}{2} k_{m} \delta_{m}^{2}=\frac{1}{2} k_{m}\left(\mathbf{V}^{T} \mathbf{q}-e\left(\Omega_{m} t\right)\right)^{2} \\
& =\frac{1}{2} k_{m} \mathbf{q}^{T} \mathbf{V} \mathbf{V}^{T} \mathbf{q}-k_{m} e\left(\Omega_{m} t\right) \mathbf{V}^{T} \mathbf{q}+\frac{1}{2} k_{m}\left[e\left(\Omega_{m} t\right)\right]^{2}
\end{aligned}
$$

Given that the strain energy relates to the shaft and/or bearing, the total strain energy can be written as

$\mathrm{U}=\mathrm{U}_{1}+\frac{1}{2} \mathbf{q}^{\mathrm{T}} \mathbf{K q}$

here

$$
\begin{aligned}
\mathbf{K} & =\left[\begin{array}{cc}
\mathbf{K}_{p} & \mathbf{0} \\
\mathbf{0} & \mathbf{K}_{g}
\end{array}\right], \\
\mathbf{K}_{i} & =\left[\begin{array}{ccccc}
k_{x i} & 0 & 0 & -k_{x i \theta_{y i}} & 0 \\
0 & k_{y i} & k_{y i \theta_{x i}} & 0 & 0 \\
0 & k_{y i \theta_{x i}} & k_{\theta_{x i}} & 0 & 0 \\
-k_{x i \theta_{y i}} & 0 & 0 & k_{\theta_{y i}} & 0 \\
0 & 0 & 0 & 0 & 0
\end{array}\right]
\end{aligned}
$$

After Lagrange equations are applied, the equation of motions for the un-damped system is derived as

$$
\mathbf{M} \ddot{\mathbf{q}}+\mathbf{G} \dot{\mathbf{q}}+\left(\mathbf{K}+k_{m} \mathbf{V} \mathbf{V}^{T}\right) \mathbf{q}+\tilde{Q}=\mathbf{F}_{i n}+\mathbf{F}_{e x}
$$

here

$$
\begin{aligned}
\tilde{Q}= & \left(0,0,-\frac{1}{2} J_{P i}\left(\ddot{\alpha}_{i} \theta_{y i}+2 \dot{\alpha}_{i} \dot{\theta}_{y i}\right), \frac{1}{2} J_{P i}\left(\ddot{\alpha}_{i} \theta_{x i}\right.\right. \\
& \left.\left.+2 \dot{\alpha}_{i} \dot{\theta}_{x i}\right), \frac{1}{2} J_{P i}\left(\theta_{x i} \ddot{\theta}_{y i}-\theta_{y i} \ddot{\theta}_{x i}\right)\right)^{T} \\
\mathbf{F}_{i n}= & {\left[\begin{array}{c}
\mathbf{F}_{i n}^{p} \\
\mathbf{F}_{i n}^{g}
\end{array}\right], \quad \mathbf{M}=\left[\begin{array}{cc}
\mathbf{M}_{p} & 0 \\
0 & \mathbf{M}_{g}
\end{array}\right], \quad \mathbf{G}=\left[\begin{array}{cc}
\mathbf{G}_{p} & 0 \\
0 & \mathbf{G}_{g}
\end{array}\right] } \\
\mathbf{F}_{i n}^{i}= & \left(m_{i} e_{i}\left(\Omega_{i}+\dot{\alpha}_{i}\right)^{2},-m_{i} e_{i} \ddot{\alpha}_{i}, 0,0,-m_{i} e_{i} \ddot{y}_{i}\right)^{T} \\
& \times \cos \left(\Omega_{i} t+\alpha_{i}+\varphi_{i}\right) \\
& +\left(m_{i} e_{i}\left(\Omega_{i}+\dot{\alpha}_{i}\right)^{2}, m_{i} e_{i} \ddot{\alpha}_{i}, 0,0, m_{i} e_{i} \ddot{x}_{i}\right)^{T} \\
& \times \sin \left(\Omega_{i} t+\alpha_{i}+\varphi_{i}\right) \\
\mathbf{F}_{e x}= & -k_{m} e\left(\Omega_{m} t\right) \mathbf{V}^{T}+\left(0,0,0,0, T_{i n p}, 0,0,0,0, T_{\text {out }}\right)^{T}
\end{aligned}
$$

The equivalent viscous damping matrix for the supporting shaft and bearing, and the linear mesh damping are introduced in a form similar to the equivalent stiffness matrix as

$\mathbf{C}=\mathbf{C}_{s}+c_{m} \mathbf{V} \mathbf{V}^{T}$

Then the equation of motions for the herringbone gear pair is derived under the form

$$
\mathbf{M} \ddot{\mathbf{q}}+(\mathbf{G}+\mathbf{C}) \dot{\mathbf{q}}+\left(\mathbf{K}+k_{m} \mathbf{V} \mathbf{V}^{T}\right) \mathbf{q}+\tilde{Q}=\mathbf{F}_{i n}+\mathbf{F}_{e x}
$$

Table 2 Natural frequency of gear

\begin{tabular}{lll}
\hline Modal no. & \multicolumn{2}{l}{ Natural frequency } \\
\cline { 2 - 3 } & $\mathrm{Rad} / \mathrm{s}$ & $\mathrm{rpm}$ \\
\hline 1 & 1323.2 & 12,636 \\
2 & 1323.2 & 12,636 \\
3 & 1649.1 & 15,748 \\
4 & 2059.0 & 19,662 \\
5 & 2245.1 & 21,439 \\
6 & 5020.5 & 47,942 \\
\hline
\end{tabular}

It should be noted that matrix $\mathbf{C}_{s}$ defines the external damping of the shaft and bearing, which can be set up by proportional damping as

$\mathbf{C}_{s}=\alpha_{1} \mathbf{M}+\beta_{1} \mathbf{K}$

Here $\alpha_{1}$ and $\beta_{1}$ refer to the supporting damping coefficients of the system and can be obtained according to the natural frequency of herringbone gear, which is listed in Table 2.

\section{Numerical results}

\subsection{Effect of damping on modal vibration}

For the convenience of numerical analysis, the second order differential equation (20) can be written as a state equation

$\dot{\mathbf{X}}=\mathbf{A X}+\mathbf{B}$

by introducing a new state variable

$\mathbf{X}=(\mathbf{q}, \dot{\mathbf{q}})^{T}$

here,

$\mathbf{A}=\left[\begin{array}{c:c}\mathbf{0} & \mathbf{I} \\ \hdashline-\mathbf{M}^{-1}\left(\mathbf{K}+k_{m} \mathbf{V} \mathbf{V}^{T}\right) & -\mathbf{M}^{-1}(\mathbf{G}+\mathbf{C})\end{array}\right]$,

$\mathbf{B}=\left[\begin{array}{c}\mathbf{0} \\ -\mathbf{M}^{-1}\left(\mathbf{F}_{i n}+\mathbf{F}_{e x}-\tilde{Q}\right)\end{array}\right]$

The resulting system of equation gives non self-adjoint eigenvalue problem. In general, the eigenvalues are a function of the rotating spin speed of the pinion shaft (in the present work, the gear reduced speed ratio is assumed to be constant) and are in the form [15] (In page 40 of the book)

$\lambda_{j}=\omega_{j}\left(\varsigma_{j} \pm k \sqrt{1-\varsigma_{j}^{2}}\right), \quad(j=1,2, \ldots N)$ 
here, $k^{2}=-1, \varsigma_{j}$ is the frequency damping constant or damping ratio, and $\omega_{j}$ is the damped natural frequency of whirl speed.

In the first case, the external damping, the mesh damping, and the eccentric mass are all ignored. The time varying mesh stiffness is assumed to be constant with mean value shown in Fig. 3. Figure 6 shows the Campbell diagram of the herringbone gear transmission system. There are two forward- whirling modes in which the pinion whirls in the direction of its spin speed, and two backward-whirling modes in which the pinion whirls opposite to the direction of its spin speed.

As for rotor dynamics, a critical speed of orders of a single shaft rotor is defined as spin speed for which a multiple of that speed coincides with one of the systems' natural frequencies. The intersection of the Synchronous Whirl Line (shortened by SWL) $\omega_{j}=s \Omega_{i}$ with the natural frequency curve $\omega_{j}$
Fig. 6 Campbell diagram and critical speed relative to shaft frequency (undamped)

Fig. 7 Campbell diagram and critical speed relative to mesh frequency (undamped)
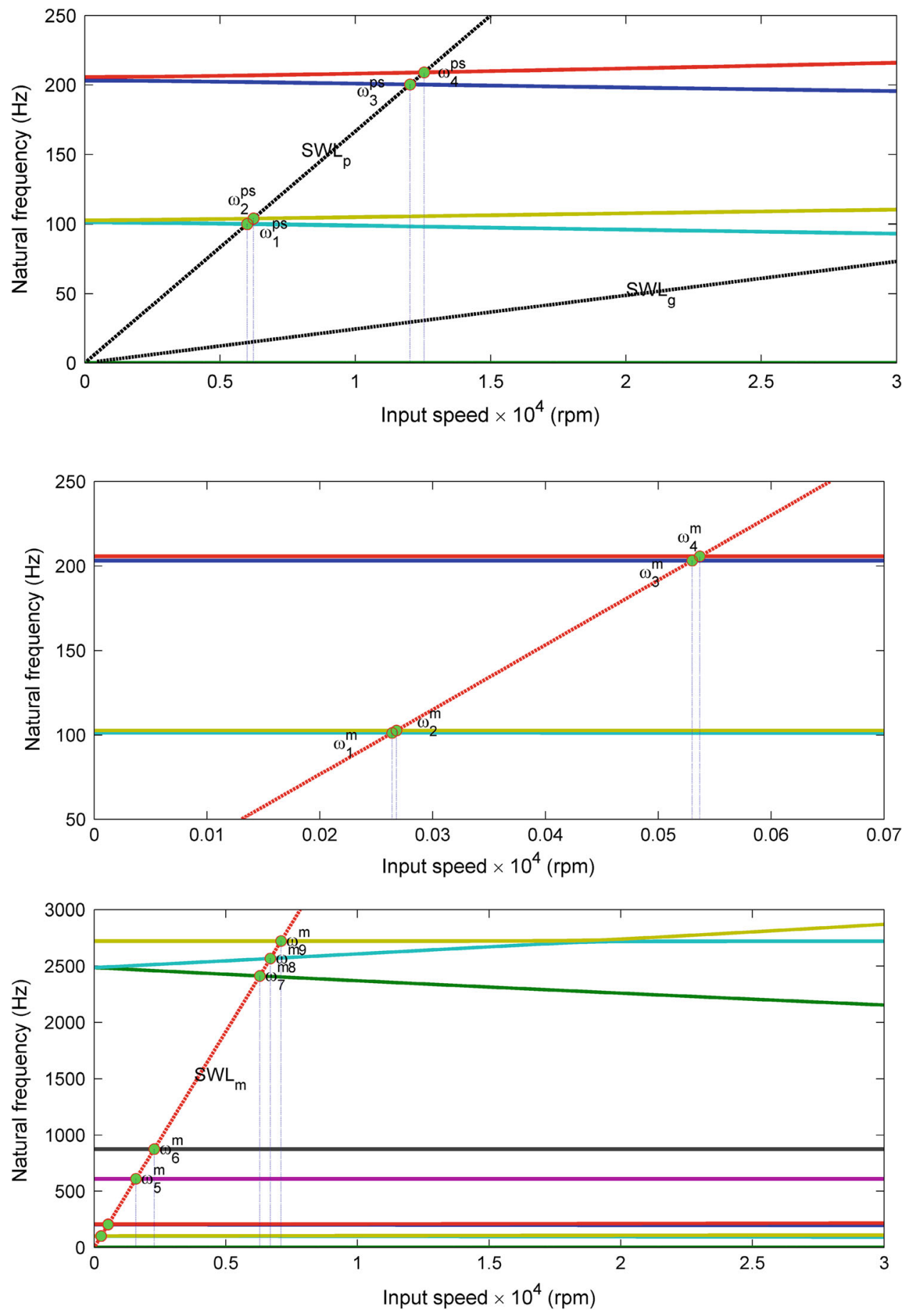
Fig. 8 Campbell diagram and critical speed relative to shaft frequency (damped)

Fig. 9 Campbell diagram and critical speed relative to mesh frequency (damped)
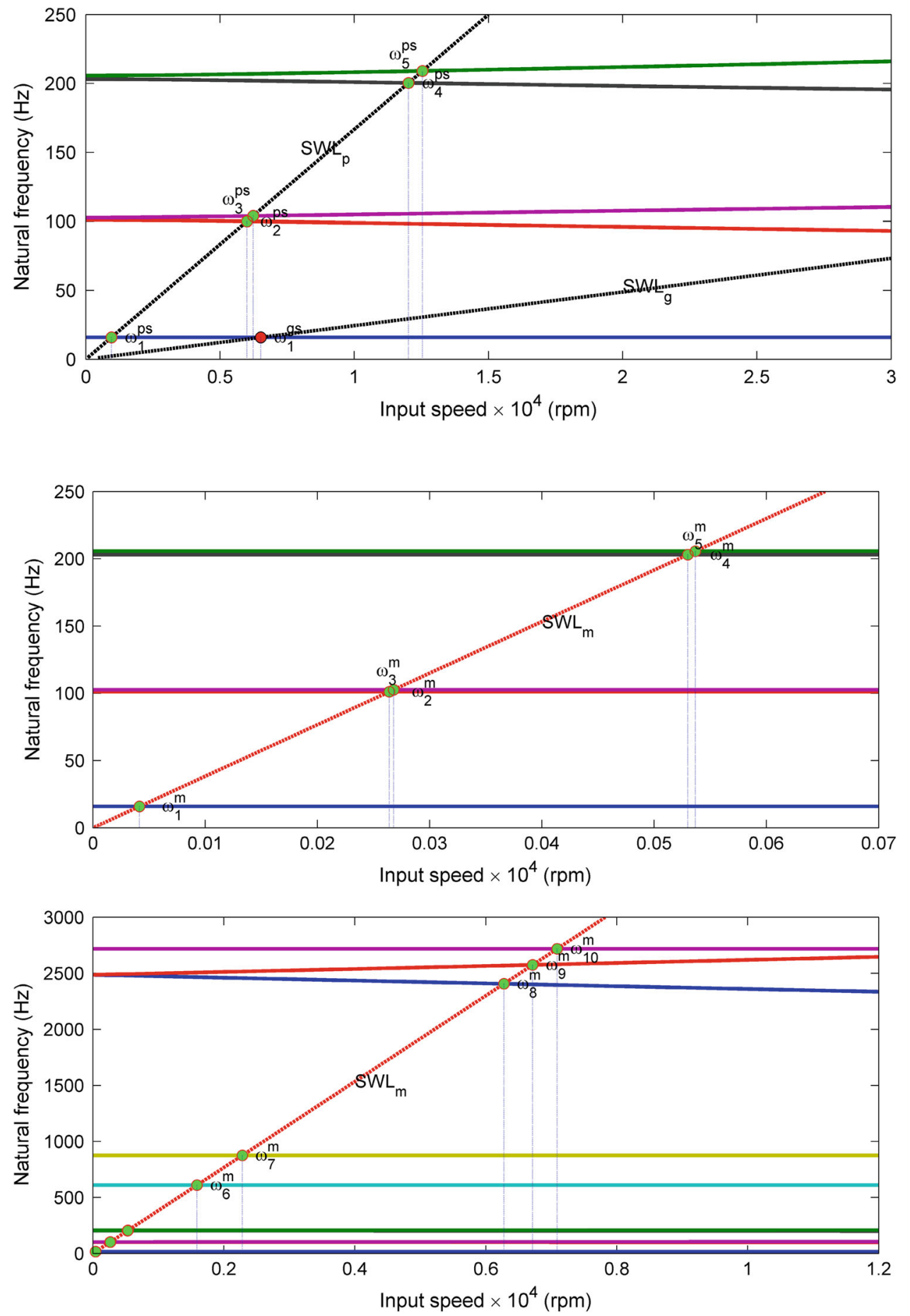

defines the critical speed. For the gear transmission system, two coupled shafts lead to two SWLs, as shown in Fig. 6. When the pinion rotates in region $(0$ and 30,000$) \mathrm{rpm}$, there are four intersection points between $\mathrm{SWL}_{\mathrm{p}}$ (the subscript $\mathrm{p}$ indicates the SWL relative to the pinion shaft speed, and $g$ is for the gear) and natural frequency curves. Points $\omega_{1}^{p s}$ and $\omega_{3}^{p s}$ are backward synchronous whirling modes while $\omega_{2}^{p s}$ and $\omega_{4}^{p s}$ are forward synchronous whirling modes. Generally, the backward synchronous whirling is exposed to stronger damping because material damping becomes active due to the alternating deformation of the shaft. When the forward synchronous whirling occurs, the gear pair rotation is dangerous as the shaft rotates in bent condition and its deformation does not produce damping. Therefore, in the present herringbone model, the critical speeds are $\omega_{2}^{p s}(\approx 6,232 \mathrm{rpm})$ and $\omega_{4}^{p s}(\approx 12,544 \mathrm{rpm})$. 
Fig. 10 Damping ratio, the circle indicates the damping ratio relative to the shaft critical speed, and the triangle is for the mesh frequency

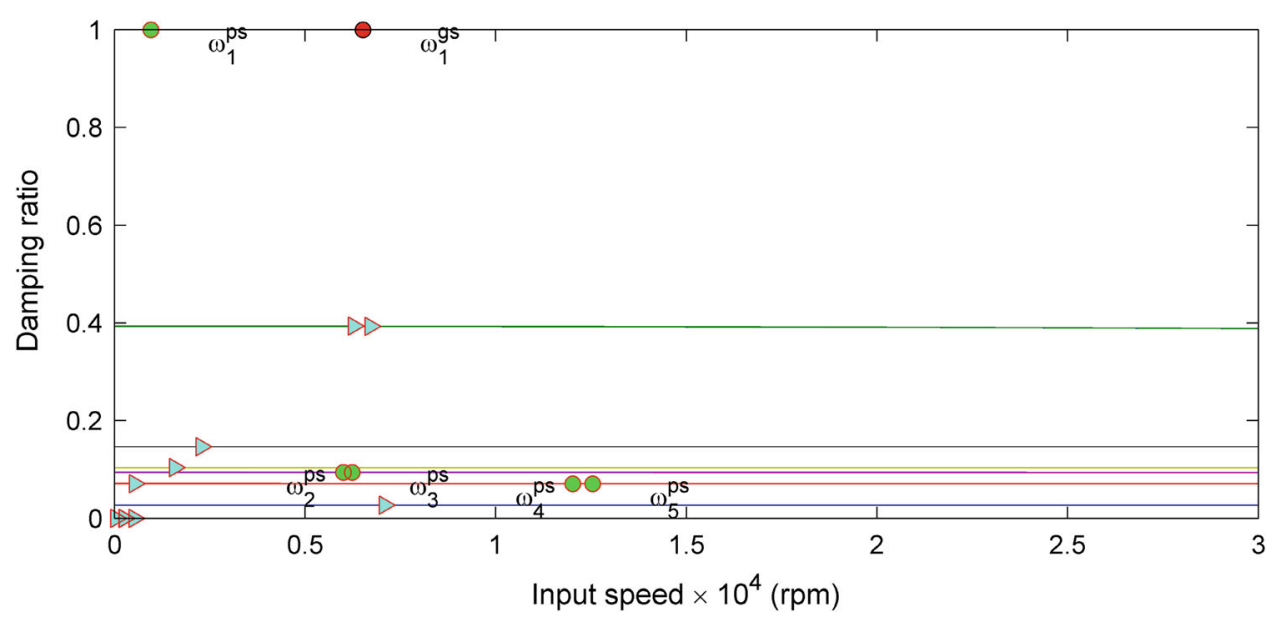

As for gear dynamics, a critical speed relating to mesh frequency is more important as the gear vibration is mainly determined by the time varying mesh stiffness and static transmission error, which are varying with mesh frequency. Figure 7 shows the Campbell diagram and $\mathrm{SWL}_{\mathrm{m}}$ (the subscript $\mathrm{m}$ is identical for mesh frequency). It is observed that the natural frequency curves intersect with $\mathrm{SWL}_{\mathrm{m}}$ nine times at $\omega_{j}^{m}(j=1,2, \ldots 9)$. During the acceleration phase, the gear transmission system will encounter four critical speeds at $\omega_{2,4,6,8}^{p s}(\approx 267.8,536.7,2281.2,6695.1 \mathrm{rpm})$. Additionally, the higher whirling frequency curves $\omega_{7}$ and $\omega_{8}$ have significant changes varying along with the pinion shaft speed.

Frequency veering phenomena [7] are observed between curve $\omega_{8}$ and $\omega_{9}$ for the pinion speed around 18,990 rpm. During the veering phenomena, frequency curves do not cross but swap their trends and maintain continuity as the speed increases. In practice, the intensive coupling between the higher whirling modes $\omega_{8}$ and $\omega_{9}$ may exist in a high-speed herringbone gear transmission system.

In the second case, the external damping and mesh damping are introduced with other parameters being the same as the first case.

The Campbell diagrams of the damped system are shown in Figs. 8 and 9. The damping ratios are shown in Fig. 10. A comparison with the Campbell diagrams in the first case indicates: (1) a lower whirling frequency curve is appearing and there are five intersection points with $\mathrm{SWL}_{\mathrm{p}}$ and one intersection point with $\mathrm{SWL}_{\mathrm{g}}$. (2) The lowest whirling frequency seems to be little affected by the spin speed. The rotation motions at the critical speed $\omega_{1}^{p s}$ and $\omega_{1}^{g s}$ are safe thanks to the high damping ratio, which is equal to one. The imagine part of eigenvalue is zero, and the amplitude of gear vibration will decay exponentially without any oscillation. (3) The resonance vibration at backward and forward synchronous whirling frequency $\omega_{8}^{m}$ and $\omega_{9}^{m}$ will be attenuated due to the high damping ratio $\varsigma_{8}=$ $\varsigma 9=0.3934$. (4) The herringbone gear system undergoes unstable motion when the input shaft speed is lower than $\Omega_{p}=536.7 \mathrm{rpm}$.

\subsection{Effect of eccentric mass on modal vibration}

In this section, the eccentric mass in the pinion and gear is considered. Let $e_{g}=0.1 \mathrm{~mm}$ and $e_{p}=0.01 \mathrm{~mm}$, and other parameters are the same as the previous section unless specified otherwise. The natural frequencies and damping ratio of the herringbone gear system with and without eccentric mass under different damping conditions are listed in Table 3.

It is observed that: (1) Under undamped condition, the eccentric mass will reduce the critical speed of the herringbone gear system, especially for modal 6 , the critical speed decreases $44 \%$. As mentioned above, the forward synchronous whirling will occurs at modal 3, 5, 7, 9. (2) Under damped condition, the trend affected by the eccentric mass is the same as that under undamped condition. Two new low critical speeds are found located at $41.519 \mathrm{rpm}$ for mesh frequency and $954.93 \mathrm{rpm}$ for shaft speed. Furthermore, the first critical speed is safe for the damping ratio is 1 . The subsequent three critical speeds are identical with zero damping ratio, which means that the herringbone gear system will oscillate with the increasing of time.

\subsection{Effect of mesh stiffness on modal vibration}

A constant drag torque $14,120 \mathrm{Nm}$ is adopted and time varying mesh stiffness calculated in Sect. 2 is used to analyze the dynamic response of the herringbone gear system. Letting input shaft speed $\Omega_{p}=954.93 \mathrm{rpm}$, the torsional response (TR, $\theta$ ) and lateral response (LR, y direction) at the pinion are obtained within 50 periods of shaft time. The FFT analysis made from time $\mathrm{t}=0$ is shown in Fig. 11, in which the red dotted line is for the linear time invariant mesh stiffness and the blue line is for the time varying case. The coupled natural frequency at $\Omega_{p}=954.93 \mathrm{rpm}$ and correspond- 
Table 3 Natural frequency and damping ratio

\begin{tabular}{|c|c|c|c|c|c|c|c|c|c|c|}
\hline & \multicolumn{4}{|c|}{ Undamped/rpm } & \multicolumn{6}{|c|}{ Damped/rpm } \\
\hline & \multicolumn{2}{|l|}{ UEC } & \multicolumn{2}{|l|}{$\mathrm{EC}$} & \multicolumn{3}{|l|}{ UEC } & \multicolumn{3}{|l|}{$\mathrm{EC}$} \\
\hline & $\mathrm{S}$ & M & $\mathrm{S}$ & M & $\mathrm{S}$ & M & $\mathrm{D}$ & $\mathrm{S}$ & M & $\mathrm{D}$ \\
\hline 1 & - & - & - & - & 954.93 & 41.519 & 1 & 954.93 & 41.519 & 1 \\
\hline 2 & 6004.5 & 264.08 & 5580.0 & 243.13 & 6004.1 & 264.08 & 0 & 5579.9 & 243.13 & 0 \\
\hline 3 & 6232.2 & 267.83 & 6173.0 & 267.82 & 6232.6 & 267.83 & 0 & 6173.1 & 267.82 & 0 \\
\hline 4 & 12025.0 & 529.88 & 11273.0 & 491.86 & 12024.0 & 529.88 & 0 & 11273.0 & 491.87 & 0 \\
\hline 5 & 12544.0 & 536.71 & 12422.0 & 536.68 & 12545.0 & 536.71 & 0.0710 & 12423.0 & 536.68 & 0.0710 \\
\hline 6 & & 1589.4 & 20679.0 & 899.04 & & 1591.0 & 0.1035 & 20692.0 & 899.62 & 0.0705 \\
\hline 7 & & 2281.2 & & 2281.2 & & 2281.2 & 0.1465 & & 2281.2 & 0.1465 \\
\hline 8 & & 6291.3 & & 5694.5 & & 6275.8 & 0.3934 & & 5691.9 & 0.0519 \\
\hline 9 & & 6695.1 & & 6293.0 & & 6715.9 & 0.3934 & & 6276.0 & 0.3934 \\
\hline 10 & & 7099.1 & & 6696.9 & & 7089.9 & 0.0270 & & 6716.1 & 0.3934 \\
\hline
\end{tabular}

Note that UEC indicates that the eccentric mass is not considered, and EC means with eccentric mass. S represents critical speed with respect to shaft speed and $\mathrm{M}$ is critical speed with respect to mesh frequency

Fig. 11 Frequency spectrum of TR and LR
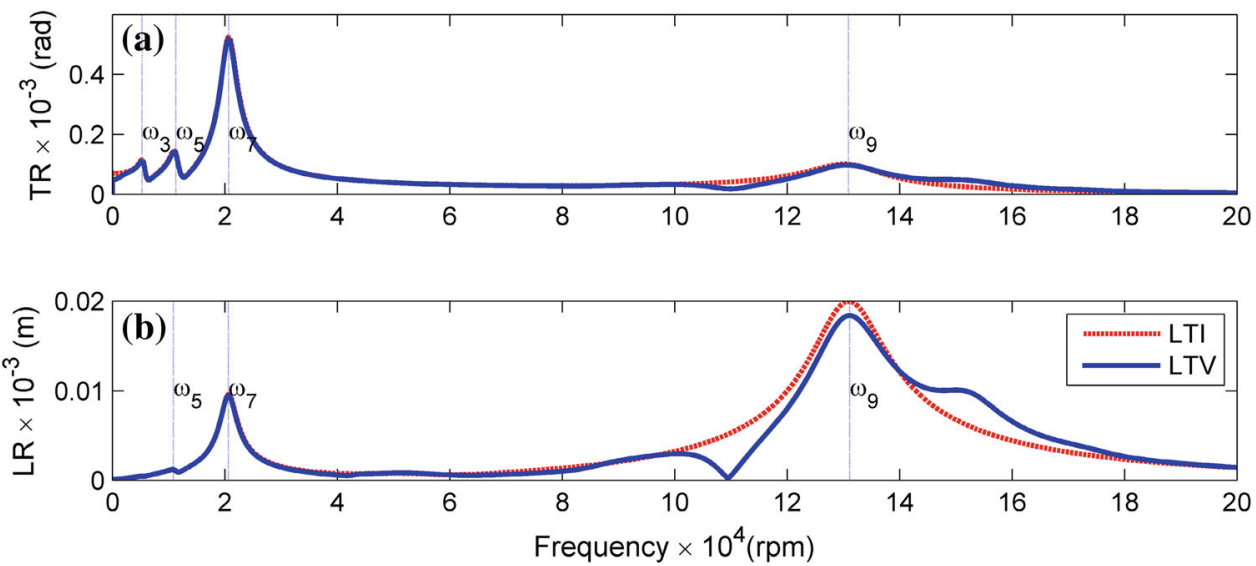

ing frequency components are also listed in Table 4. It is indicated that the LR has shown frequency component to be $10853,20635,131123 \mathrm{rpm}$ and the TR has shown four predominant frequencies 5274, 11310, 20687, $130918 \mathrm{rpm}$. It is noted that the frequency component at 5,274 rpm of TR is faintness. In both cases, the response is predominated by the higher frequency components, which coincides with the situation that the modal damping is 1 at this input speed case. And the high frequency component may be a result of frequency veering phenomena as shown in Figs. 7 and/or 9. When the input speed is low, the mesh frequency as a predominant frequency will be close to it. The severity is prominent at the gear pair, which is harmful to the running of the machine.

When the time varying stiffness is added to the original system, the frequency response is shown in Fig. 11. It is seen that the time varying mesh stiffness may reduce the ampli-
Table 4 Coupled natural frequency and response spectra in rpm with constant mesh stiffness

\begin{tabular}{lclll}
\hline \multicolumn{5}{c}{$\Omega_{p}=954.93 \mathrm{rpm}$} \\
& Natural frequency & Damping ratio & LR & TR \\
\hline 1 & 0 & - & - & - \\
2 & 954.93 & 1 & & \\
3 & 5591.7 & 0.1000 & & 5,274 \\
4 & 6160.3 & 0.0936 & & \\
5 & 11313.0 & 0.0715 & 10,853 & 11,310 \\
6 & 12344.0 & 0.0710 & & \\
7 & 20691.0 & 0.0705 & 20,635 & 20,687 \\
8 & 52467.0 & 0.1465 & & \\
9 & 130911.0 & 0.0519 & 131,123 & 130,918 \\
10 & 148411.0 & 0.3936 & & \\
11 & 149900.0 & 0.3936 & & \\
\hline
\end{tabular}


tude of high order frequency around 130,918 rpm obviously. Hence, in high-speed applications of a gear pair, it is much more useful to acquire the critical speed relative to mesh frequency than that relative to shaft speed. It may be noted that the current analysis is limited, since the static time transmission error is not included and the transient analysis is performed.

\section{Conclusions}

The analytical model for a herringbone geared system was proposed considering the coupling of lateral and torsional motion. Firstly, the equivalent mesh stiffness of the herringbone gear pair is obtained based on the finite element method considering the rim and web. Then, the modal property of vibration characteristics and transient behaviors in high-speed condition were investigated with the gyroscopic effect. Finally, natural frequencies and corresponding damping ratio of the coupled system, acting as a function of the spin speed of the input shafts, were first determined for different damping and eccentric mass conditions. The results, which were presented by means of Campbell diagram, were helpful in explaining the effects of gear mesh mechanisms especially in high-speed applications.

In addition, synchronous whirling motions of the herringbone gear pair were analyzed. The results show that the damping affects the critical speed slightly while the eccentric mass will reduce the critical speed significantly. Transient dynamics analysis was also performed with time invariant mesh stiffness and time varying mesh stiffness. In both cases, the high frequency components are predominant, which may be the results of frequency veering phenomena at high order natural frequency. The results indicate that it is necessary to pay attention to the critical speed relative to mesh frequency in high-speed gear applications.

Acknowledgments The authors gratefully acknowledge the support of the National Science Foundation of China (NSFC) through Grants Nos. 51304562, 51275530, National Key Basic Research Program of China (2011CB706800).

\section{References}

1. Anderson NE, Nightingale L, Wagner DA (1989) Design and test of a propfan gear system. J Propuls Power 5:95-102

2. Kahraman A (1994) Planetary gear train dynamics. J Mech Des 116:713-720

3. Parker RG (2012) Vibration properties of high-speed planetary gears with gyroscopic effects. J Vib Acoust 134:061014
4. Ericson TM, Parker RG (2013) Planetary gear modal vibration experiments and correlation against lumped-parameter and finite element models. J Sound Vib 332:2350-2375

5. Lee AS, Ha JW, Choi D-H (2003) Coupled lateral and torsional vibration characteristics of a speed increasing geared rotor-bearing system. J Sound Vib 263:725-742

6. Kang MR, Kahraman A (2012) Measurement of vibratory motions of gears supported by compliant shafts. Mech Syst Signal Process 29:391-403

7. Chouksey M, Dutt JK, Modak SV (2012) Modal analysis of rotorshaft system under the influence of rotor-shaft material damping and fluid film forces. Mech Mach Theory 48:81-93

8. Lund JW (1978) Critical speeds, stability and response of a geared train of rotors. J Mech Des 100:535-538

9. Iida H, Tamura A, Kikuchi K, Agata H (1980) Coupled torsionalflexural vibration of a shaft in a geared system of rotors: 1st report. Bull JSME 23:2111-2117

10. Kahraman A, Ozguven HN, Houser DR, Zakrajsek JJ (1992) Dynamic analysis of geared rotors by finite elements. J Mech Des 114:507-514

11. Baud S, Velex P (2002) Static and dynamic tooth loading in spur and helical geared systems-experiments and model validation. J Mech Des 124:334-346

12. Baguet $S$, Velex $P(2005)$ Influence of the nonlinear dynamic behavior of journal bearings on gear-bearing assemblies. ASME 5:735745

13. Baguet $S$, Jacquenot $G$ (2010) Nonlinear couplings in a gear-shaftbearing system. Mech Mach Theory 45:1777-1796

14. Kang CH, Hsu WC, Lee EK, Shiau TN (2011) Dynamic analysis of gear-rotor system with viscoelastic supports under residual shaft bow effect. Mech Mach Theory 46:264-275

15. Friswell MI, Penny JE, Garvey SD, Lees AW (2010) Dynamics of rotating machines. Cambridge University Press, Cambridge

16. Velex P, Ajmi M (2006) On the modelling of excitations in geared systems by transmission errors. J Sound Vib 290:882-909

17. Ajmi M, Velex P (2005) A model for simulating the quasi-static and dynamic behaviour of solid wide-faced spur and helical gears. Mech Mach Theory 40:173-190

18. Abousleiman V, Velex P, Becquerelle S (2007) Modeling of spur and helical gear planetary drives with flexible ring gears and planet carriers. J Mech Des 129:95

19. Kumar KS, Rao BS (2012) Coupled lateral-torsonal vibration analysis of a geared shaft rotor system using a complex rotor variable approach. Int J Acoust Vib 17:127-132

20. Kuma KS, Rao BS (2012) An experimental study of whirling characteristics of gear-pinion rotor system. Int J Mech Appl 2:10-13

21. White G (1998) Design study of a split-torque helicopter transmission. Proc Inst Mech Eng G 212:117-123

22. Bu Z, Liu G, Wu L (2012) Modal analyses of herringbone planetary gear train with journal bearings. Mech Mach Theory 54:99-115

23. Ajmi M, Velex P (2001) A model for simulating the quasi-static and dynamic behavior of double helical gears. MPT ... Fukuoka: the JSME international conference on motion and power transmissions, pp 132-137

24. Palermo A, Mundo D, Hadjit R, Desmet W (2013) Multibody element for spur and helical gear meshing based on detailed threedimensional contact calculations. Mech Mach Theory 62:13-30

25. Velex P, Ajmi M (2007) Dynamic tooth loads and quasi-static transmission errors in helical gears: approximate dynamic factor formulae. Mech Mach Theory 42:1512-1526

26. Muszynska A (2005) Rotordynamics. Taylor \& Francis, Boca Roton 